\title{
Revisión sistemática de evaluaciones económicas de los sistemas de telemonitorización en los marcapasos
}

\author{
Antonio López-Villegass ${ }^{\mathrm{a}, \mathrm{b}, *}$, Daniel Catalán-Matamoros ${ }^{\mathrm{c}}$, Carlos Martín-Saborido ${ }^{\mathrm{d}}$, \\ Irene Villegas-Tripiana ${ }^{e}$ y Emilio Robles-Musso ${ }^{\mathrm{f}}$
}

a Division of Medicine, Nordland Hospital, Bodø, Noruega

${ }^{\mathrm{b}}$ Institute of Clinical Medicine, Faculty of Health Sciences, University of Tromsø, Tromsø, Noruega

${ }^{\mathrm{c}}$ Departamento de Periodismo y Comunicación, Universidad Carlos III, Madrid, España

${ }^{\mathrm{d}}$ Unidad de Evaluación de Tecnología Sanitaria, Universidad Francisco de Vitoria, Madrid, España

e Unidad de Apoyo a la Investigación y Biblioteca, Hospital de Poniente, El Ejido, Almería, España

${ }^{\mathrm{f}}$ Unidad de Cuidados Intensivos, Hospital de Poniente, El Ejido, Almería, España

Historia del artículo:

Recibido el 9 de marzo de 2015

Aceptado el 8 de junio de 2015

On-line el 21 de octubre de 2015

\section{Palabras clave:}

Estudios de seguimiento

Gasto cardiaco

Marcapasos

\section{RES U M E N}

Introducción y objetivos: En la última década, la telemedicina aplicada a la monitorización de marcapasos cardiacos ha experimentado un extraordinario crecimiento. Se desconoce si esta tecnología tiene una eficiencia diferente de la convencional. El objetivo del estudio es realizar una revisión sistemática analizando la evidencia disponible con respecto al consumo de recursos y los resultados en salud en ambas modalidades de seguimiento.

Métodos: La búsqueda se realizó en 11 bases de datos y se incluyeron estudios publicados hasta noviembre de 2014. Los criterios de inclusión fueron: a) diseño experimental u observacional; b) estudios basados en evaluaciones económicas completas; c) pacientes con marcapasos, y d) telemonitorización comparada con la modalidad hospitalaria.

Resultados: Siete estudios cumplían los criterios de inclusión, con información sobre 2.852 pacientes con una media de edad de 81 años; el bloqueo auriculoventricular era la principal indicación. En la telemonitorización, los eventos cardiovasculares se detectan y tratan 2 meses antes, con lo que se reduce en un 34\% el número de hospitalizaciones, así como las visitas rutinarias y de urgencias al hospital. No hubo diferencias intergrupales significativas en calidad de vida percibida o número de eventos adversos. El coste de la telemonitorización es un 60\% menor que el de la monitorización hospitalaria.

Conclusiones: En la telemonitorización de marcapasos, los eventos cardiovasculares se detectan antes y disminuyen las hospitalizaciones y el número de visitas al hospital. Además, con la telemonitorización se reducen los costes asociados al seguimiento.

(c) 2015 Sociedad Española de Cardiología. Publicado por Elsevier España, S.L.U. Todos los derechos reservados.

\section{A Systematic Review of Economic Evaluations of Pacemaker Telemonitoring Systems}

A B S T R A C T

\section{Keywords:}

Follow-up studies

Cardiac output

Pacemaker
Introduction and objectives: Over the last decade, telemedicine applied to pacemaker monitoring has undergone extraordinary growth. It is not known if telemonitoring is more or less efficient than conventional monitoring. The aim of this study was to carry out a systematic review analyzing the available evidence on resource use and health outcomes in both follow-up modalities.

Methods: We searched 11 databases and included studies published up until November 2014. The inclusion criteria were: $a$ ) experimental or observational design; $b$ ) studies based on complete economic evaluations; $c$ ) patients with pacemakers, and $d$ ) telemonitoring compared with conventional hospital monitoring.

Results: Seven studies met the inclusion criteria, providing information on 2852 patients, with a mean age of 81 years. The main indication for device implantation was atrioventricular block. With telemonitoring, cardiovascular events were detected and treated 2 months earlier than with conventional monitoring, thus reducing length of hospital stay by $34 \%$ and reducing routine and emergency hospital visits as well. There were no significant intergroup differences in perceived quality of life or number of adverse events. The cost of telemonitoring was $60 \%$ lower than that of conventional hospital monitoring.

\footnotetext{
* Autor para correspondencia: Division of Medicine, Nordland Hospital, Prinsens Gate 164, Postbox 1480 (n. ${ }^{\circ} 38$ ), 8005 Bodø, Noruega. Correo electrónico: antoniolopezvillegas@andaluciajunta.es (A. López-Villegas).
} 
Conclusions: Compared with conventional monitoring, cardiovascular events were detected earlier and the number or hospitalizations and hospital visits was reduced with pacemaker telemonitoring. In addition, the costs associated with follow-up were lower with telemonitoring.

Full English text available from: www.revespcardiol.org/en

(c) 2015 Sociedad Española de Cardiología. Published by Elsevier España, S.L.U. All rights reserved.

\section{Abreviaturas}

MH: monitorización hospitalaria

TM: telemonitorización

\section{INTRODUCCIÓN}

La enfermedad cardiovascular es una de las principales causas de morbimortalidad y motiva el $30 \%$ de la mortalidad global, según la Organización Mundial de la Salud ${ }^{1}$. El actual envejecimiento de la población y el incremento de la esperanza de vida son factores que influyen en la incidencia de las enfermedades cardiovasculares, y en ocasiones es necesario que los pacientes con este tipo de enfermedades precisen de dispositivos electrónicos cardiovasculares implantables. El número de dispositivos electrónicos cardiovasculares implantables -en el que se agrupan marcapasos, desfibriladores automáticos implantables, terapias de resincronización cardiaca y Holters- continúa creciendo a un ritmo exponencial desde que se realizara el primer implante ${ }^{2}$ en 1958. Un marcapasos es un dispositivo electrónico diseñado para producir impulsos eléctricos con el objeto de estimular el corazón cuando falla la estimulación fisiológica o normal ${ }^{3}$. Entre otras razones, el crecimiento del número de marcapasos implantados en la última década ha llevado a la saturación de las consultas de cardiología ${ }^{4,5}$.

La telemonitorización (TM) consiste en el uso de equipos electrónicos para observar o registrar procesos fisiológicos mientras el paciente realiza actividades de la vida diaria. Se refiere a la medición a distancia de procesos fisiológicos tales como signos vitales (frecuencia cardiaca, respiración, presión arterial) y otras medidas (células sanguíneas, bioquímica hemática, producción renal, etc.) usando tecnología digital y analógica ${ }^{6}$. Esta tecnología tuvo su origen en la década de los setenta, con la monitorización transtelefónica ${ }^{7}$, y a principios del siglo xxi con la llegada del primer marcapasos que se puede monitorizar a distancia utilizando cable y/ o banda ancha ${ }^{8}$. La monitorización transtelefónica tenía la capacidad de proporcionar información básica, como el aviso del inminente agotamiento de la batería, pero no notificaba problemas en el funcionamiento del dispositivo o el control de los parámetros programados erróneamente. La llegada de la monitorización a distancia ha permitido el acceso a gran cantidad de información, con la ventaja de que los profesionales sanitarios pueden consultarla en cualquier momento. El desarrollo y la expansión de la TM de marcapasos ha generado la necesidad de realizar estudios que muestren su eficiencia respecto a la monitorización hospitalaria $(\mathrm{MH})$. Por lo tanto, el objetivo de este estudio es realizar una revisión sistemática de evaluaciones económicas para analizar la evidencia disponible sobre el consumo de recursos y resultados en salud en ambas modalidades de seguimiento.

\section{MÉTODOS}

\section{Estrategia de búsqueda}

La búsqueda se realizó el 1 de diciembre de 2014, sin limitaciones en cuanto al idioma o el año de publicación. Las bases de datos utilizadas fueron MEDLINE (a través de PubMed),
EMBASE, DARE, HTA y NHS Economic Evaluation Database (NHS EED), LILACS, IME y CUIDEN y las tesis doctorales incluidas en Teseo, TDR y Dialnet, así como literatura gris: actas de congresos, libros y publicaciones académicas. Además, se realizaron búsquedas manuales de las referencias bibliográficas consideradas de interés e incluidas en revisiones sistemáticas y metanálisis. Los operadores booleanos utilizados fueron AND y OR. Se utilizaron los siguientes términos de búsqueda en inglés: pacemaker, telemedicine, remote consultation, home monitoring, cost-benefit analysis. Los descriptores/palabras clave utilizados para la búsqueda de artículos en español fueron: marcapasos, telemedicina, consulta remota, monitorización domiciliaria, análisis de coste-beneficio. La búsqueda de los términos o palabras clave se realizó en el artículo completo en cada una de las bases de datos seleccionadas en la revisión, incluyéndose título, resumen, texto, palabras clave, etc. Los criterios de inclusión de estudios fueron: $a$ ) diseño experimental u observacional; b) estudios basados en evaluaciones económicas completas, es decir, estudios donde se compararon los resultados en salud y costes, sin excluir ninguna modalidad de análisis (costeefectividad, coste-utilidad, coste-beneficio y minimización de costes); d) pacientes con marcapasos, y d) TM comparada con MH.

\section{Extracción de datos}

Dos investigadores (Antonio López-Villegas e Irene Villegas-Tripiana) realizaron en el mes de diciembre de 2014 y de manera independiente la extracción y la lectura de todos los títulos y resúmenes de los estudios seleccionados inicialmente a través de la estrategia de búsqueda (tabla 1). En función de los objetivos del estudio, se preseleccionaron los artículos que potencialmente reunían los criterios de inclusión. Al mes siguiente, los mismos investigadores realizaron la lectura a texto completo de los artículos cribados previamente. En los casos en que no hubo consenso sobre la inclusión/exclusión de un artículo, un tercer investigador (Daniel Catalán-Matamoros) realizó la mediación. Las variables incluidas en el análisis de datos fueron: a) características del estudio (autor, año de publicación, país, duración del estudio, tamaño de la muestra, edad, sexo, principal indicación para el implante y marcapasos utilizados), y $b$ ) análisis y principales resultados de las variables (análisis realizado, variables principales, variables secundarias, resultados de salud y resultados de costes). Dos revisores (Carlos Martín-Saborido y Emilio Robles-Musso) valoraron independientemente la calidad metodológica de los

Tabla 1

Estrategia de búsqueda realizada en MEDLINE (a través de PubMed)

\begin{tabular}{ll}
\hline & Términos de búsqueda \\
\hline$\# 1$ & Search (pacemaker) OR pacemakers \\
\hline$\# 2$ & Search telemedicine \\
\hline$\# 3$ & Search remote consultation \\
\hline$\# 4$ & Search home monitoring \\
\hline$\# 5$ & Search (((cost-benefit analysis) OR cost benefit analysis) \\
& OR cost benefit) OR cost-benefit \\
\hline$\# 6$ & $\# 1$ and \#2 or \#3 or \#4 \\
\hline$\# 8$ & $\# 1$ and \#5 \\
\hline
\end{tabular}


artículos seleccionados utilizando como herramienta de evaluación la lista de comprobación de López-Bastida et al $^{9}$.

\section{RESULTADOS}

Se identificó en la búsqueda un total de 1.730 artículos. Después de revisar a texto completo 108 estudios potencialmente relevantes, siete artículos ${ }^{10-16}$ cumplían los criterios de selección (figura) y se incluyeron en la posterior síntesis de la evidencia. Las referencias de los 101 artículos excluidos se pueden consultar en el material suplementario.

\section{Características de los estudios seleccionados}

Se incluyeron tres estudios experimentales y cuatro de diseño descriptivo/observacional, con el objetivo de evaluar los resultados en calidad de vida, eficacia, seguridad, fiabilidad y costes de la TM de marcapasos en comparación con la $\mathrm{MH}^{10-16}$.

Las principales características de los estudios se resumen en la tabla 2. Los estudios seleccionados representan un total de 2.876 pacientes incluidos, 1.303 de ellos en ensayos clínicos aleatorizados. Las muestras estudiadas son variables (96-802 pacientes). La media de edad en cinco de los estudios ${ }^{12-16}$ era $81,40 \pm 6,77$ años (intervalo de confianza del 95\%, 73,00-89,80). La principal indicación para el implante del marcapasos fue el bloqueo auriculoventricular ${ }^{12-16}$. El periodo de estudio varía entre $10 \mathrm{y}$ 80 meses. En todos los trabajos seleccionados se utilizó el mismo modelo de marcapasos en ambos brazos de seguimiento, excepto en los estudios de Folino et al ${ }^{14,15}$ donde la MH se llevó a cabo utilizando dos modelos diferentes de marcapasos. En ninguno de los estudios seleccionados se comenta si los sistemas de monitorización estaban

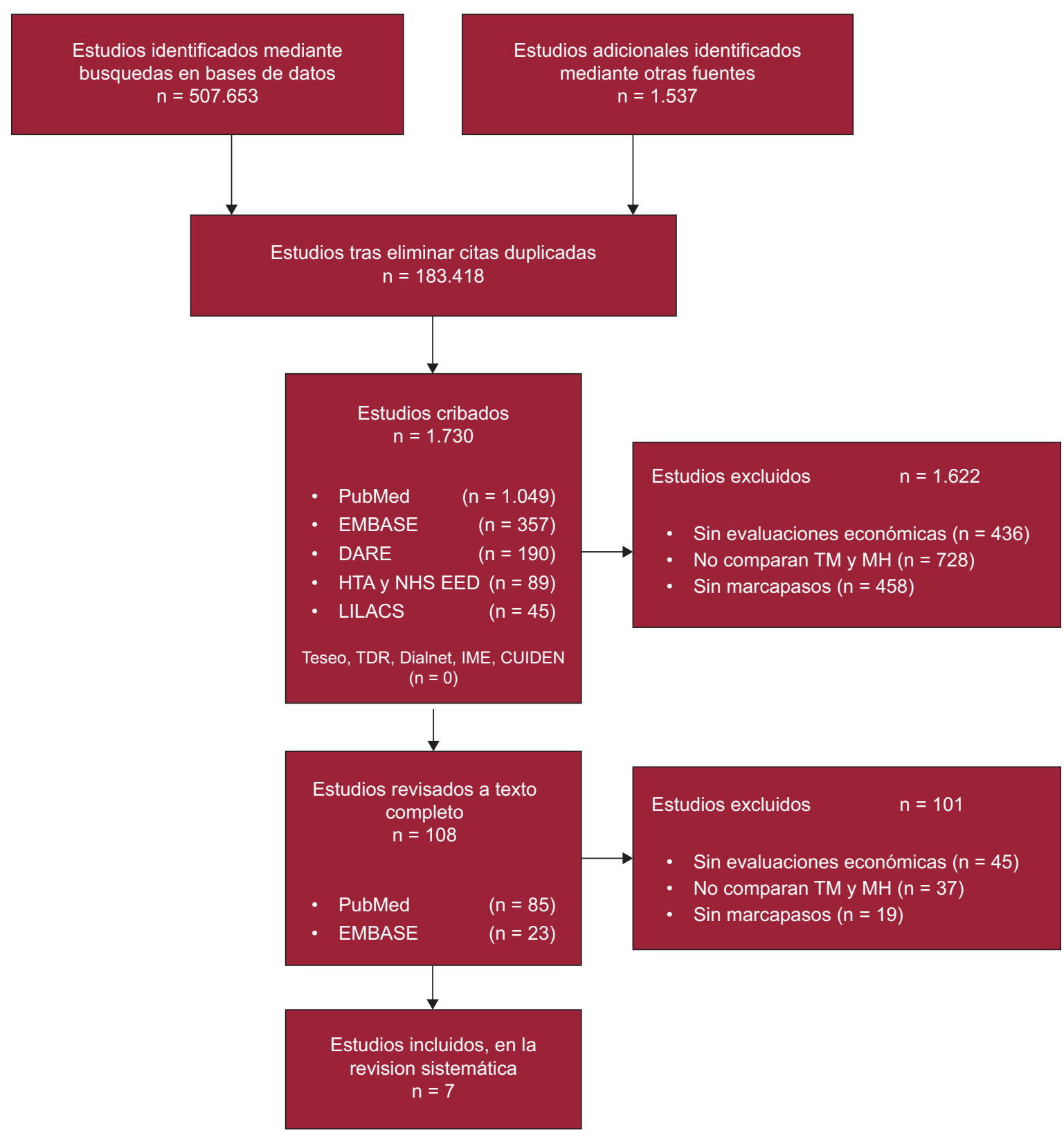

Figura. Diagrama de flujo PRISMA del proceso de selección de estudios para la revisión sistemática de evaluaciones económicas de los sistemas de telemonitorización y seguimiento de marcapasos. MH: monitorización hospitalaria; TM: telemonitorización. 


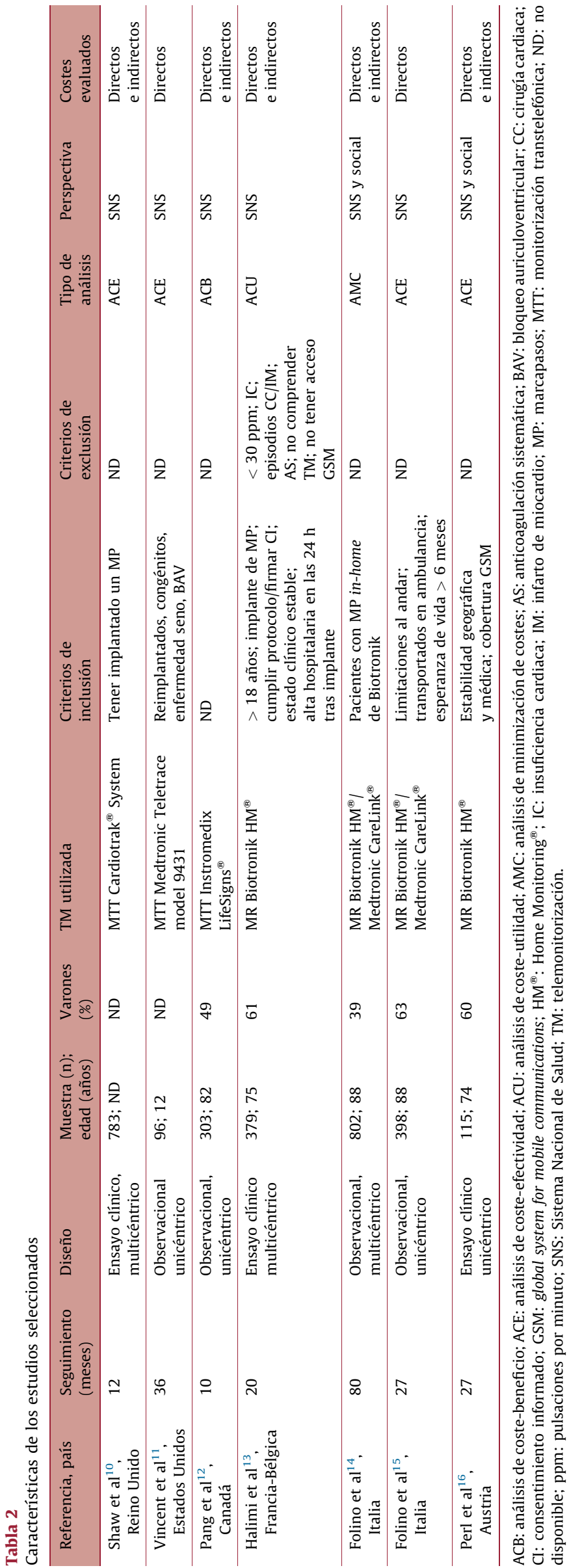

utilizándose previamente para todos los marcapasos controlados por el hospital.

\section{Resultados del análisis de las variables de salud}

La tabla 3 recoge las variables principales y secundarias analizadas en cada uno de los estudios, así como los resultados más significativos. En cuatro publicaciones se realiza un análisis de coste-efectividad $^{10,11,15,16}$. El número de recambios de marcapasos varía entre 7 y 123 implantados ${ }^{14,15}$, mientras que la vida útil es de 6,7 a 8,3 años ${ }^{12-15}$. Solo en el estudio de Halimi et al ${ }^{13}$ se especifica la estancia hospitalaria media, que fue un 34\% más corta en el grupo de TM, y además es el único estudio en el que se evaluó la calidad de vida mediante el cuestionario SF-36, que no encontró diferencias significativas entre ambos grupos.

En el estudio de Pang et al $^{12}$ se pone de manifiesto que, del total de las transmisiones de emergencia en el grupo de TM, solo en el $8 \%$ se detectaron eventos adversos, lo que se incrementa hasta el $52 \%$ en el estudio de Folino et al $^{15}$. Los pacientes del grupo de TM que asistieron al hospital para la reprogramación del marcapasos ${ }^{14,15}$ fueron un 0,6-1,9\%. En el estudio OEDIPE ${ }^{13}$, el 20,1\% de los pacientes del grupo de TM tuvieron un evento adverso frente al 19,0\% del grupo hospitalario. La mortalidad anual ${ }^{12-15}$ fue de un $0-11,7 \%$.

\section{Análisis de costes}

El importe de los costes de implantación de la TM no se indica en ninguno de los estudios seleccionados. Vincent et $\mathrm{al}^{11}$ señalan que los costes los asume el hospital, mientras que en los artículos presentados por Folino et al ${ }^{14,15}$ los fabricantes de los marcapasos asumían los costes de la TM. Con el objetivo de facilitar la comparación entre las diferentes monedas incluidas en las publicaciones seleccionadas, en primer lugar se calculó la inflación anual acumulada desde el año siguiente a la publicación del artículo hasta diciembre de 2014, y luego se realizó una conversión directa de cada moneda a euros en función de la cotización del 20 de febrero de 2015. Los siete estudios incluidos en la revisión presentan unos costes de TM menores que los de la MH (tabla 3). El ahorro para el Sistema Nacional de Salud asociado al transporte en ambulancia se estimó en el estudio West-Scotland ${ }^{10}$ en 13.594 euros anuales al sustituir un sistema de seguimiento por otro. La estimación se realizó para los 637 pacientes pertenecientes a tres hospitales de Ayrshire, de los 783 incluidos en el estudio West-Scotland ${ }^{10}$. El estudio de Vincent et $\mathrm{al}^{11}$ indica que se habría producido un ahorro de 17.247 euros en los 3 años que duró la investigación si los 96 pacientes incluidos en el estudio hubieran sustituido las visitas a la sala de emergencia por el sistema de transmisión de datos. Las diferencias en el impacto económico se hacen patentes en varios estudios $^{12,13}$ cuyas diferencias entre los costes de la TM pueden llegar a ser entre 4 y 8 veces inferiores a los hallados en la opción hospitalaria. Los pacientes del grupo TM realizaron de media dos visitas menos al hospital (TM frente a MH, 5,92 frente a 7,1 ${ }^{13}$. Folino et $\mathrm{al}^{14}$ estimaron una diferencia media del $20,5 \%$ en los costes entre ambos grupos; además, en ese mismo estudio, si los pacientes tenían que desplazarse al hospital en ambulancia, la diferencia aumentaba hasta el 66,5\%. En otros estudios se presentan los resultados separados en función del tipo de coste/año; por ejemplo, en el SAVE-HM ${ }^{16}$, los costes de personal fueron significativamente más bajos en el grupo TM $(18,0 \pm 41,3$ frente a 22,4 $\pm 26,9$ euros; $\mathrm{p}<0,003)$; igualmente, se incluyen los costes informales asociados al transporte utilizado por los pacientes para asistir al hospital, y se muestra que, en relación con los kilómetros recorridos, los costes absolutos del grupo de TM son menores que en el hospitalario en casi un $60 \%$ ( 872,14 frente a $2.162,78$ euros). En la tabla 4 se incluyen los costes asociados a cada modalidad de seguimiento. 
Tabla 3

Análisis y principales resultados de las variables evaluadas

\begin{tabular}{|c|c|c|c|c|c|c|c|c|c|c|c|c|c|c|c|}
\hline \multirow[t]{2}{*}{ Referencia, país } & \multirow[t]{2}{*}{$\begin{array}{l}\text { Variables } \\
\text { principales }\end{array}$} & \multirow[t]{2}{*}{$\begin{array}{l}\text { Variables } \\
\text { secundarias }\end{array}$} & \multicolumn{2}{|c|}{$\begin{array}{c}\mathrm{N} \cdot{ }^{\circ} \mathrm{de} \\
\text { hospitalizaciones }\end{array}$} & \multicolumn{2}{|c|}{$\begin{array}{l}\text { Seguimientos/ } \\
\text { paciente/año }\end{array}$} & \multicolumn{2}{|c|}{$\begin{array}{l}\text { Eventos } \\
\text { adversos/año }\end{array}$} & \multicolumn{2}{|c|}{$\begin{array}{l}\text { Visitas a } \\
\text { urgencias }\end{array}$} & \multicolumn{2}{|c|}{ Mortalidad anual } & \multicolumn{2}{|c|}{$\begin{array}{l}\text { Análisis de costes/año, } \\
\text { moneda original/euros }\end{array}$} & \multirow[t]{2}{*}{ Conclusiones } \\
\hline & & & $\mathrm{MH}$ & TM & $\mathrm{MH}$ & $\mathrm{TM}$ & $\mathrm{MH}$ & $\mathrm{TM}$ & $\mathrm{MH}$ & TM & $\mathrm{MH}$ & TM & $\mathrm{MH}$ & TM & \\
\hline $\begin{array}{l}\text { Shaw et al }{ }^{10} \\
\text { Reino Unido }\end{array}$ & $\begin{array}{l}\text { Costes del } \\
\text { transporte TM }\end{array}$ & $\begin{array}{l}\text { Efectividad y } \\
\text { costes sanitarios }\end{array}$ & ND & 1 & ND & ND & ND & 1 & ND & 1 & $\begin{array}{l}3,7 \% \text { fallecidos } \\
\text { entre ambos } \\
\text { grupos }\end{array}$ & & $\begin{array}{l}\text { Ahorro anual } \\
10.000 £ / 13.5\end{array}$ & $\begin{array}{l}\text { en transporte: } \\
94,30 €\end{array}$ & $\begin{array}{l}\text { El éxito alcanzado permitirá expandir } \\
\text { el servicio a otros hospitales }\end{array}$ \\
\hline $\begin{array}{l}\text { Vincent et } \mathrm{al}^{11} \text {, } \\
\text { Estados Unidos }\end{array}$ & $\begin{array}{l}\text { Eficacia de } \\
\text { la TM }\end{array}$ & $\begin{array}{l}\text { Costes directos } \\
\text { de la TM }\end{array}$ & ND & ND & ND & 4,76 & ND & $1 \%$ & ND & ND & $\mathrm{ND}$ & ND & $\begin{array}{l}\text { TM supone un } \\
\text { de } 19.000 \$ / 1\end{array}$ & $\begin{array}{l}\text { ahorro anual } \\
7.247,48 €\end{array}$ & $\begin{array}{l}\text { La TM fue significativamente efectiva } \\
\text { correlacionando con la presencia o } \\
\text { ausencia de problemas del MP }\end{array}$ \\
\hline $\begin{array}{l}\text { Pang et al al } \\
\text { Canadá }\end{array}$ & $\begin{array}{l}\text { Eficacia y } \\
\text { viabilidad } \\
\text { de la TM }\end{array}$ & $\begin{array}{l}\text { Extrapolar los } \\
\text { costes de la MH } \\
\text { a la TM }\end{array}$ & ND & ND & ND & 4,7 & $4,1 \%$ & $5,3 \%$ & ND & ND & $\begin{array}{l}12 \text { fallecidos } \\
\text { entre ambos } \\
\text { grupos }\end{array}$ & & $\begin{array}{l}88.230 \$ 1 \\
78.038,21 €\end{array}$ & $\begin{array}{l}11.744 \$ / \\
10.387,40 €\end{array}$ & $\begin{array}{l}\text { La TM es segura, permite el } \\
\text { seguimiento a pacientes con } \\
\text { dificultades y reduce costes }\end{array}$ \\
\hline $\begin{array}{l}\text { Halimi et al }^{13} \\
\text { Francia-Bélgica }\end{array}$ & $\begin{array}{l}\text { Proporción } \\
\text { de EAM }\end{array}$ & $\begin{array}{l}\text { Hospitalización, } \\
\text { costes y calidad } \\
\text { de vida }\end{array}$ & 4,8 & 3,2 & 7,1 & 5,92 & $19,0 \%$ & $20,1 \%$ & ND & ND & 1 & 0 & $7.414 €$ & $7.125 €$ & $\begin{array}{l}\text { La TM es segura y facilita el } \\
\text { seguimiento de pacientes }\end{array}$ \\
\hline $\begin{array}{l}\text { Folino et al }{ }^{14} \text {, } \\
\text { Italia }\end{array}$ & Eficacia & $\begin{array}{l}\text { Costes sanitarios } \\
\text { e informales }\end{array}$ & ND & ND & ND & 0,45 & ND & 0,3 & ND & ND & $\begin{array}{l}8,7 \% \text { entre } \\
\text { ambos grupos }\end{array}$ & & $68,43 €$ & $56,77 €$ & $\begin{array}{l}\text { La TM es tan segura y fiable como la } \\
\text { MH. Los costes de la TM fueron el } \\
20,5 \% \text { más bajos que los de la MH }\end{array}$ \\
\hline $\begin{array}{l}\text { Folino et al }{ }^{15} \text {, } \\
\text { Italia }\end{array}$ & $\begin{array}{l}\text { Longevidad, } \\
\text { ECG y datos } \\
\text { tecnicos del MP }\end{array}$ & $\begin{array}{l}\text { Impacto } \\
\text { económico de la } \\
\text { TM }\end{array}$ & ND & ND & 1,3 & 2,6 & ND & $52 \%$ & ND & ND & $8,3 \%$ & $11,7 \%$ & $73,80 €$ & $37,26 €$ & $\begin{array}{l}\text { La TM es segura y coste-efectiva y } \\
\text { detecta episodios de arritmias } \\
\text { tempranos }\end{array}$ \\
\hline $\begin{array}{l}\text { Perl et al }{ }^{16} \text {, } \\
\text { Austria }\end{array}$ & $\begin{array}{l}\text { Costes y número } \\
\text { de visitas al } \\
\text { hospital }\end{array}$ & $\begin{array}{l}\text { Seguridad de la } \\
\text { TM }\end{array}$ & 15 & 11 & 0,53 & 0,29 & $\begin{array}{l}\text { Sin } \\
\text { diferer } \\
\text { signifi }\end{array}$ & $\begin{array}{l}\text { icias } \\
\text { ativas }\end{array}$ & ND & ND & ND & ND & $\begin{array}{l}\text { La TM es el } 5 \\
\text { económica qu }\end{array}$ & $\begin{array}{l}3,7 \% \text { más } \\
\text { e la MH }\end{array}$ & $\begin{array}{l}\text { La TM es segura, detecta eventos y } \\
\text { reduce el número de visitas } \\
\text { hospitalarias }\end{array}$ \\
\hline
\end{tabular}

EAM: eventos adversos mayores: ECG: electrocardiograma; MH: monitorización hospitalaria; MP: marcapasos; ND: no disponible; TM: telemonitorización. 
Tabla 4

Costes incluidos en cada modalidad de seguimiento

\begin{tabular}{|c|c|c|}
\hline Referencia, país & Telemonitorización & Monitorización hospitalaria \\
\hline Shaw et $\mathrm{al}^{10}$, Reino Unido & $\begin{array}{l}\text { Personal } \\
\text { Teléfono } \\
\text { Transporte }\end{array}$ & $\begin{array}{l}\text { Personal } \\
\text { Teléfono } \\
\text { Transporte }\end{array}$ \\
\hline Vincent et $\mathrm{al}^{11}$, Estados Unidos & $\begin{array}{l}\text { Coste mensual visitas de rutina y urgencias } \\
\text { (incluyendo análisis del MP) } \\
\text { Coste sala de urgencias sin análisis del MP }\end{array}$ & $\begin{array}{l}\text { Coste mensual visitas de rutina y urgencias } \\
\text { (incluyendo análisis del MP) } \\
\text { Coste sala de urgencias sin análisis del MP }\end{array}$ \\
\hline Pang et $\mathrm{al}^{12}$, Canadá & $\begin{array}{l}\text { Personal (enfermeras) } \\
\text { Visitas al hospital } \\
\text { Alquiler del equipo } \\
\text { Llamadas de teléfono }\end{array}$ & $\begin{array}{l}\text { Personal (médico + enfermera) } \\
\text { Servicios del hospital } \\
\text { Dietas } \\
\text { Costes de transporte }\end{array}$ \\
\hline Halimi et al $^{13}$, Francia-Bélgica & $\begin{array}{l}\text { Personal } \\
\text { Laboratorio } \\
\text { Costes indirectos (médicos y paramédicos) } \\
\text { Transporte }\end{array}$ & $\begin{array}{l}\text { Personal } \\
\text { Laboratorio } \\
\text { Costes indirectos (médicos y paramédicos) } \\
\text { Transporte }\end{array}$ \\
\hline Folino et $\mathrm{al}^{14}$, Italia & $\begin{array}{l}\text { Costes sanitarios (médico, enfermera y transporte) } \\
\text { Costes informales (transporte y productividad) } \\
\text { SNS (costes revisión MP) }\end{array}$ & $\begin{array}{l}\text { Costes sanitarios (médico, enfermera y transporte) } \\
\text { Costes informales (transporte y productividad) } \\
\text { SNS (costes chequeo MP) }\end{array}$ \\
\hline Folino et $\mathrm{al}^{15}$, Italia & $\begin{array}{l}\text { SNS (costes de la visita) } \\
\text { Personal (médico + enfermera) }\end{array}$ & $\begin{array}{l}\text { SNS (costes de la visita) } \\
\text { Personal (médico + enfermera) } \\
\text { Transporte }\end{array}$ \\
\hline Perl et $\mathrm{al}^{16}$, Austria & $\begin{array}{l}\text { Personal } \\
\text { Transporte }\end{array}$ & $\begin{array}{l}\text { Personal } \\
\text { Transporte }\end{array}$ \\
\hline
\end{tabular}

MP: marcapasos; SNS: Sistema Nacional de Salud.

\section{Evaluación de la calidad metodológica}

Las variables evaluadas se califican con respuestas de sí o no en función de la presencia o ausencia del criterio analizado (tabla 5). Si al realizar la revisión completa del artículo no se encontraba algún parámetro, se introducía la respuesta "no» en la tabla, refiriéndose a que el estudio no incluía ese parámetro.

Dos estudios ${ }^{14,16}$ obtuvieron la puntuación global más alta en calidad metodológica, 17 de los 25 puntos potenciales. La puntuación más baja fue la alcanzada por el estudio de Shaw et $\mathrm{al}^{10}$, con 7 puntos. Las publicaciones evaluadas obtuvieron una media de $12,71 \pm 4,72$ puntos (intervalo de confianza del $95 \%$, $8,35-17,08)$. Los principales hallazgos fueron:

- En dos artículos ${ }^{14,16}$ se incluyeron las perspectivas social y sanitaria.

- En cinco estudios se menciona claramente la fuente ${ }^{12-16}$ y en cuatro, el diseño y los métodos utilizados para la obtención de los datos de efectividad ${ }^{13-16}$.

- Ningún estudio aplicó técnicas de modelización o descuentos para costes y beneficios ni se realizó ningún tipo de análisis de sensibilidad.

- De cuatro publicaciones ${ }^{12-14,16}$ se puede extraer conclusiones sobre la extrapolación de los resultados a otros contextos.

- En tres artículos ${ }^{14-16}$, los resultados de costes y efectos en la salud de ambas alternativas se presentan por separado; en cambio, las limitaciones de los trabajos no se presentan de manera crítica y transparente en tres estudios ${ }^{10,11,14}$.

- Las conclusiones de todas las publicaciones ${ }^{10-16}$ responden a la pregunta de investigación.

- Las tasas de coste-efectividad y coste-beneficio no se incluyeron en ninguno de los estudios seleccionados.

\section{DISCUSIÓN}

Los hallazgos de esta revisión revelan que la TM de marcapasos, respecto a la $\mathrm{MH}$, no presenta diferencias significativas en calidad de vida relacionada con la salud y número de eventos cardiovasculares. Los resultados muestran que con la TM estos episodios se detectan y se tratan antes, lo que conduce a una reducción en el número de hospitalizaciones y visitas sistemáticas y urgentes al hospital. Además, los costes de seguimiento son inferiores a los de la MH. Transporte, personal sanitario, ausencias laborales y cuidados informales influyen en gran medida en que el impacto económico de esta tecnología varíe entre los países y las regiones donde se realizaron los estudios.

\section{Eficacia y seguridad clínica de la telemonitorización}

Los estudios incluidos en la revisión muestran que con la TM se ha producido una reducción en el número de visitas de seguimiento hospitalarias ${ }^{13,14,16}$. Estos resultados son similares a los publicados en el estudio COMPAS $^{17}$, que mostró una reducción del 55\% en el número de visitas que los pacientes con marcapasos realizaron al hospital.

El desarrollo y la expansión de la TM de marcapasos ha permitido probar que es una tecnología segura y fiable ${ }^{10-16}$. La detección temprana de eventos cardiovasculares y/o alteraciones en el funcionamiento del dispositivo ha facilitado que se trate precozmente a los pacientes, con lo que se reduce significativamente el número y la importancia de las hospitalizaciones ${ }^{18-20}$. Crossley et $\mathrm{al}^{21}$, en un estudio realizado durante 12 meses sobre 897 pacientes con marcapasos, mostraron que tales eventos se detectan 2 meses antes en el grupo de TM (5,7 frente a 7,7 meses). En dos estudios posteriores ${ }^{18,19}$, realizados durante 15 y 12 meses respectivamente, en pacientes con desfibrilador automático implantable y terapia de resincronización cardiaca, el tiempo de respuesta ante estos episodios fue 22-36 días en el caso de la $\mathrm{MH}$, mientras que en el grupo domiciliario se redujo hasta los 2,0-4,6 días. Los estudios ECOST $^{20}$ y EVATEL ${ }^{22}$ mostraron que no había diferencias significativas entre ambas modalidades de seguimiento en el número de eventos adversos detectados, coincidiendo con los resultados obtenidos en tres publicaciones de esta revisión ${ }^{13,15,16}$.

La calidad de vida es un parámetro analizado solo en el estudio de Halimi et $\mathrm{al}^{13}$, y muestra que no hubo diferencias significativas entre ambos brazos de seguimiento. Estos resultados coinciden con los de los estudios $\operatorname{COMPAS}^{17}$ y $\operatorname{ECOST}^{20}$, que utilizaron el 
Tabla 5

Lista de comprobación de la calidad metodológica de los estudios

\begin{tabular}{|c|c|c|c|c|c|c|c|}
\hline & $\begin{array}{l}\text { Shaw } \\
\text { et al }{ }^{10} \\
1981\end{array}$ & $\begin{array}{l}\text { Vincent } \\
\text { et al }{ }^{11} \\
1997\end{array}$ & $\begin{array}{l}\text { Pang } \\
\text { et } \text { al }^{12} \\
2010\end{array}$ & $\begin{array}{l}\text { Halimi } \\
\text { et } \text { al }^{13} \\
2008\end{array}$ & $\begin{array}{l}\text { Folino } \\
\text { et } \text { al }^{14} \\
2012\end{array}$ & $\begin{array}{l}\text { Folino } \\
\text { et } \text { al }^{15} \\
2013\end{array}$ & $\begin{array}{l}\text { Perl } \\
\text { et } \text { al }^{16} \\
2013\end{array}$ \\
\hline ¿El estudio establece claramente su objetivo y la pregunta de investigación? & Sí & Sí & Sí & Sí & Sí & Sí & Sí \\
\hline $\begin{array}{l}\text { ¿La evaluación económica se efectúa de modo general y posteriormente, si los } \\
\text { datos lo permiten, en subgrupos de población (edad, sexo, gravedad, } \\
\text { niveles de riesgo) en caso de que haya diferencias relevantes en la } \\
\text { efectividad o en los costes entre ellos? }\end{array}$ & No & No & Sí & Sí & Sí & Sí & Sí \\
\hline $\begin{array}{l}\text { ¿La evaluación económica incluye tanto la perspectiva social como la } \\
\text { del financiador (SNS)? }\end{array}$ & No & No & No & Sí & Sí & Sí & Sí \\
\hline ¿Se presentan ambas perspectivas de manera separada y diferenciada? & No & No & No & No & Sí & No & Sí \\
\hline ¿Se compara la tecnología al menos con la práctica clínica habitual? & Sí & Sí & Sí & Sí & Sí & Sí & Sí \\
\hline ¿Se justifica claramente la elección de la opción a comparar? & Sí & No & Sí & Sí & Sí & Sí & Sí \\
\hline $\begin{array}{l}\text { ¿Se justifica razonablemente el tipo de análisis elegido en relación con } \\
\text { la cuestión planteada? }\end{array}$ & No & No & No & Sí & Sí & Sí & Sí \\
\hline $\begin{array}{l}\text { ¿Se detalla con precisión la fuente utilizada para la obtención de datos } \\
\text { de eficacia o efectividad? }\end{array}$ & No & No & Sí & Sí & Sí & Sí & Sí \\
\hline ¿Se detallan con precisión el diseño y los métodos utilizados? & No & No & No & Sí & Sí & Sí & Sí \\
\hline $\begin{array}{l}\text { ¿Las medidas de resultados seleccionadas son clínicamente relevantes } \\
\text { (medida de eficacia/efectividad final)? }\end{array}$ & No & Sí & Sí & Sí & Sí & Sí & Sí \\
\hline $\begin{array}{l}\text { ¿Las escalas sociales de valoración de la calidad de vida relacionada con } \\
\text { la salud han sido validadas a partir de una muestra representativa de } \\
\text { la población? }\end{array}$ & No & No & No & Sí & No & No & No \\
\hline ¿Los costes recogidos se ajustan a la perspectiva de análisis seleccionada? & Sí & Sí & Sí & Sí & Sí & Sí & Sí \\
\hline $\begin{array}{l}\text { ¿Se han separado y se detallan adecuadamente las unidades físicas de } \\
\text { los costes y los datos de costes? }\end{array}$ & Sí & Sí & No & No & Sí & Sí & Sí \\
\hline $\begin{array}{l}\text { ¿El horizonte temporal considerado es el más adecuado para captar todos } \\
\text { los efectos diferenciales de las tecnologías evaluadas en la salud y los } \\
\text { recursos empleados? }\end{array}$ & Sí & Sí & Sí & Sí & Sí & Sí & Sí \\
\hline $\begin{array}{l}\text { En caso de aplicar técnicas de modelización, ¿se justifican y son transparentes } \\
\text { la elección del modelo utilizado y los parámetros y supuestos clave de este? }\end{array}$ & No & No & No & No & No & No & No \\
\hline $\begin{array}{l}\text { ¿Se descuentan los costes y resultados que se producen en el futuro } \\
\text { empleando la misma tasa? }\end{array}$ & No & No & No & No & No & No & No \\
\hline ¿Se realizó un análisis de sensibilidad? & No & No & Sí & No & No & No & No \\
\hline $\begin{array}{l}\text { ¿Se justificaron los parámetros clave del estudio y la distribución } \\
\text { estadística de las variables analizadas en el análisis de sensibilidad? }\end{array}$ & No & No & No & No & No & No & No \\
\hline $\begin{array}{l}\text { En caso de incorporar argumentos de justicia social en la evaluación (análisis } \\
\text { de equidad), ¿se presenta este análisis de forma desagregada del caso } \\
\text { principal y son transparentes los argumentos empleados? }\end{array}$ & No & No & No & No & No & No & No \\
\hline $\begin{array}{l}\text { ¿El informe permite extraer conclusiones sobre la transferibilidad o } \\
\text { extrapolación de sus resultados a otros contextos? }\end{array}$ & No & No & Sí & Sí & Sí & No & Sí \\
\hline $\begin{array}{l}\text { ¿Se presentan los resultados mediante un análisis incremental y a su vez } \\
\text { de forma desagregada (costes y resultados de las alternativas)? }\end{array}$ & No & No & No & No & Sí & Sí & Sí \\
\hline $\begin{array}{l}\text { ¿Se presentan de manera crítica y transparente las limitaciones o puntos } \\
\text { débiles del análisis? }\end{array}$ & No & No & Sí & Sí & No & Sí & Sí \\
\hline $\begin{array}{l}\text { ¿Las conclusiones del estudio responden a la pregunta de investigación } \\
\text { y se desprenden claramente de los resultados obtenidos? }\end{array}$ & Sí & Sí & Sí & Sí & Sí & Sí & Sí \\
\hline ¿Se indica quién encarga, apoya o financia el estudio? & Sí & No & No & Sí & No & No & No \\
\hline ¿Se informa sobre los posibles conflictos de intereses? & No & No & No & Sí & Sí & Sí & No \\
\hline
\end{tabular}

No: ausencia del criterio; Sí: presencia del criterio; SNS: Sistema Nacional de Salud.

cuestionario SF-36, y con un estudio recientemente publicado ${ }^{23}$ que utilizó el cuestionario EQ-5D.

La evaluación de la calidad metodológica de los estudios seleccionados mostró una gran heterogeneidad entre ellos, y los estudios más recientes obtuvieron una puntuación más alta. Los resultados mostrados en esta revisión muestran la dificultad existente en evaluar con criterios actuales la calidad metodológica de estudios realizados en décadas previas ${ }^{10,11}$. Aun así, hay ciertos parámetros que se muestran coincidentes en todos los estudios seleccionados, como un objetivo y una pregunta de investigación preestablecidas, comparación de ambas modalidades de seguimiento, ajuste de los costes recogidos a la perspectiva del análisis seleccionada y adaptación del horizonte temporal a los objetivos del estudio; por otro lado, también coinciden en no aplicar técnicas de modelización, descontar costes, realizar análisis de sensibilidad, justificar parámetros clave y distribución estadística de las variables, realizar análisis de equidad e incluir los cocientes de coste-efectividad y coste-beneficio.

\section{Aspectos económicos de la telemonitorización}

El seguimiento de pacientes con enfermedades cardiovasculares supone no solo una carga muy importante para las personas que las presentan, sino que, además, conlleva una cantidad 
importante de recursos económicos procedentes de los diferentes sistemas nacionales de salud.

Los resultados presentados en el estudio OEDIPE ${ }^{13}$ mostraron que la TM redujo un 50\% las estancias hospitalarias. Aun así, los costes totales no presentaron diferencias significativas respecto al seguimiento hospitalario, debido al sistema de reembolso basado en grupos relacionados con el diagnóstico.

La información proporcionada en esta revisión confirma que esta tecnología, debido a la reducción en el número de visitas y hospitalizaciones, presenta un claro potencial para reducir los costes sanitarios asociados tanto al personal (profesionales de la salud y administrativos) como a otros aspectos relacionados con el seguimiento (costes de transporte, mantenimiento de las consultas, etc.). Estos resultados son similares a los hallados en otras evaluaciones económicas realizadas con desfibriladores automáticos implantables ${ }^{19,24,25}$. Raatikainen et $\mathrm{al}^{24}$ mostraron que, al reducirse el número de visitas al hospital, los costes por paciente se reducían en un $41 \%$ ( 524 euros). Este ahorro aumentaba hasta el $60,9 \%$ en el estudio de Elsner et al $^{26}$, que estimó un ahorro anual de 712,31 euros/paciente gracias a un descenso del $63,2 \%$ tanto del número de visitas como de los costes de transporte asociados a estas. En un estudio posterior realizado por Crossley et $\mathrm{al}^{18}$, las estancias hospitalarias de los pacientes se redujeron un $18 \%$ en el caso de la TM, que supuso un ahorro de 1.659 dólares por paciente y año. Fauchier et al $^{27}$ estimaron en 2.149 euros/paciente el ahorro económico que suponía la TM en los 5 años que los desfibriladores automáticos implantables tienen de vida útil.

\section{Limitaciones y futuras líneas de investigación}

Este análisis presenta varias limitaciones. La primera es el escaso número de estudios seleccionados $(\mathrm{n}=7)$ y participantes incluidos $(\mathrm{n}=2.852)$, principalmente porque el uso de la tecnología de TM es escasa en comparación con la MH. La segunda limitación se halla en la heterogeneidad metodológica de los estudios seleccionados, puesto que no se utilizaron técnicas de modelización y descuentos en los costes y resultados ni se justificaron los parámetros clave del estudio y la distribución estadística de las variables analizadas en el análisis de sensibilidad. La tercera limitación consiste en la inexistencia de ensayos clínicos que estimen los resultados a medio y largo plazo, fundamentalmente debido a que se trata de una tecnología relativamente nueva. La cuarta limitación es la diferencia de 32 años entre los estudios seleccionados; en ese tiempo los cambios tecnológicos han sido enormes, y en este estudio se puede comprobar las similitudes y diferencias entre una y otra tecnología, que se han utilizado en contextos espaciotemporales diferentes. Finalmente, los estudios de coste-efectividad, en comparación con los de efectividad, son menos generalizables, puesto que dependen tanto de la duración como del contexto en el que se lleven a cabo. Aun así, su importancia es enorme, puesto que facilitan la toma de decisiones de los diferentes agentes implicados. Esta es la primera revisión sistemática de evaluaciones económicas en la que se analizan los resultados en salud y consumo de recursos asociados a la TM de pacientes con marcapasos. El estudio permitió actualizar el estado actual de conocimiento en dicha materia describiendo los resultados obtenidos en ambos brazos de seguimiento y ofrecer así herramientas para la futura toma de decisiones y nuevas políticas de salud.

En futuras investigaciones se aconseja la realización de evaluaciones económicas que comparen ambas modalidades de monitorización, incluyendo los cocientes de coste-efectividad $\mathrm{y}$ los costes informales asociados al seguimiento. El horizonte temporal debería ser a medio y largo plazo.

Este estudio se puede utilizar tanto por los gestores sanitarios como por los profesionales de los servicios de cardiología para promover la sostenibilidad de los sistemas sanitarios actuales.

\section{CONCLUSIONES}

En la TM de marcapasos los eventos cardiovasculares se detectan antes y disminuyen las hospitalizaciones y el número de visitas al hospital. Además, se reducen los costes asociados al seguimiento.

\section{FINANCIACIÓN}

Este proyecto se ha financiado parcialmente por la European Economic Area Grant 008/ABELCM/2014A de la convocatoria de investigación NILS Science and Sustainability Program 2014.

\section{CONFLICTO DE INTERESES}

Ninguno.

\section{MATERIAL SUPLEMENTARIO}

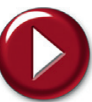

Se puede consultar material suplementario a este artículo en su versión electrónica disponible en doi:10.1016/j. recesp.2015.06.021.

\section{BIBLIOGRAFIA}

1. Tarride JE, Lim M, DesMeules M, Luo W, Burke N, O'Reilly D, et al. A review of the cost of cardiovascular disease. Can J Cardiol. 2009;25:e195-202.

2. Dubner S, Auricchio A, Steinberg JS, Vardas P, Stone P, Brugada J, et al. ISHNE/ EHRA expert consensus on remote monitoring of cardiovascular implantable electronic devices (CIEDs). Europace. 2012;14:278-93.

3. Marcapasos. ¿En qué consiste un marcapasos? Madrid: Fundación Española del Corazón; 2012 [citado 10 Abr 2015]. Disponible en: http://www. fundaciondelcorazon.com/informacion-para-pacientes/tratamientos/ marcapasos.html.

4. Osca J, Sancho Tello MJ, Navarro J, Cano O, Raso R, Castro JE, et al. Fiabilidad técnica y seguridad clínica de un sistema de monitorización remota de dispositivos cardiacos antiarrítmicos. Rev Esp Cardiol. 2009;62:886-95.

5. Al-Razzo O, Gonzáles-Villegas E, Silvestre-García J, Cabestrero-Alonso D, Represa-Pastor T, Alejandre-Leyva M, et al. Seguimiento domiciliario como sustitutivo de seguimiento presencial en pacientes portadores de marcapasos. Cuadernos de Estimulación Cardiaca. 2011;4:57-62.

6. Monitorización remota (telemonitorización). Sevilla: Observatorio de Prácticas Innovadoras en el Manejo de Enfermedades Crónicas Complejas, Consejería de Igualdad, Salud y Políticas Sociales, Junta de Andalucía; 2015 [citado 22 Feb 2015]. Disponible en: http://www.opimec.org/glosario/remote-monitoring/

7. Burri $\mathrm{H}$, Heidbüchel $\mathrm{H}$, Jung W, Brugada P. Remote monitoring: a cost or an investment? Europace. 2011;13 Suppl 2:44-8.

8. Louis AA, Turner T, Gretton M, Baksh A, Cleland JG. A systematic review of telemonitoring for the management of heart failure. Eur J Heart Fail. 2003:5:583-90.

9. López-Bastida J, Oliva J, Antoñanzas F, García-Altés A, Gisbert R, Mar J, et al. Propuesta de guía para la evaluación económica aplicada a las tecnologías sanitarias. Gac Sanit. 2010;24:154-70.

10. Shaw GB, Evans AL, Brewster GM, Groden BM, Murdoch WR. Telephone monitoring of patients with pacemakers in the west of Scotland. Br Med J (Clin Res Ed). 1981;283:127-9.

11. Vincent JA, Cavitt DL, Karpawich PP. Diagnostic and cost effectiveness of telemonitoring the pediatric pacemaker patient. Pediatr Cardiol. 1997;18:86-90.

12. Pang HW, Campbell D, Hopman WM, Brennan FJ, Abdollah H, Redfearn DP, et al Effectiveness and feasibility of a transtelephonic monitoring program: implications for a time of crisis. Int J Cardiol. 2010;145:529-30.

13. Halimi F, Clémenty J, Attuel P, Dessenne X, Amara W; OEDIPE trial Investigators. Optimized post-operative surveillance of permanent pacemakers by home monitoring: the OEDIPE trial. Europace. 2008:10:1392-9.

14. Folino AF, Breda R, Calzavara P, Migliore F, Iliceto S, Buja G. In-home controls of pacemakers in debilitated elderly patients. Geriatr Gerontol Int. 2012;12:30-5

15. Folino AF, Breda R, Calzavara P, Borghetti F, Comisso J, Iliceto S, et al. Remote follow-up of pacemakers in a selected population of debilitated elderly patients. Europace. 2013;15:382-7.

16. Perl S, Stiegler P, Rotman B, Prenner G, Lercher P, Anelli-Monti M, et al. Socioeconomic effects and cost saving potential of remote patient monitoring (SAVE-HM trial). Int J Cardiol. 2013;169:402-7.

17. Mabo P, Victor F, Bazin P, Ahres S, Babuty D, Da Costa A, et al. A randomized trial of long-term remote monitoring of pacemaker recipients (the COMPAS trial). Eur Heart J. 2012;33:1105-11. 
18. Crossley GH, Boyle A, Vitense H, Chang Y, Mead RH; CONNECT Investigators. The CONNECT (Clinical Evaluation of Remote Notification to Reduce Time to Clinical Decision) trial: the value of wireless remote monitoring with automatic clinician alerts. J Am Coll Cardiol. 2011;57:1181-9.

19. Varma N, Epstein AE, Irimpen A, Schweikert R, Love C; TRUST Investigators. Efficacy and safety of automatic remote monitoring for implantable cardioverter-defibrillator follow-up: the Lumos-T Safely Reduces Routine Office Device Follow-up (TRUST) trial. Circulation. 2010;122:325-32.

20. Guédon-Moreau L, Lacroix D, Sadoul N, Clémenty J, Kouakam C, Hermida JS, et al; ECOST trial Investigators. A randomized study of remote follow-up of implantable cardioverter defibrillators: safety and efficacy report of the ECOST trial. Eur Heart J. 2013;34:605-14.

21. Crossley GH, Chen J, Choucair W, Cohen TJ, Gohn DC, Johnson WB, et al; PREFER Study Investigators. Clinical benefits of remote versus transtelephonic monitoring of implanted pacemakers. J Am Coll Cardiol. 2009;54:2012-9.

22. Mabo P, Sadoul N, Davy JM, Deharo JC, Kacet S, Bellissant E, et al. EVATEL: Remote Follow-up of Patients Implanted with an ICD: the Prospective randomized EVATEL study. ESC Congress report, presentado en el European Society of Cardiology Congress 2011, París, Francia, 27-31 de Agosto de 2011 [citado 4 Jun 2015]. Disponible en: http://www.escardio.org/Congresses-\&-Events/
Congress-resources/ESC-Congress-365/ESC-Congress/Session-Reports/EVATELRemote-follow-up-of-patients-implanted-with-an-ICD-the-prospective-rand

23. López-Villegas A, Catalán-Matamoros D, Robles-Musso E, Peiró S. Efectividad comparativa del seguimiento remoto a personas con marcapasos cardíacos frente al convencional: calidad de vida a los 6 meses. Rev Esp Salud Pública. 2015;89:149-58.

24. Raatikainen MJ, Uusimaa P, Van Ginneken MM, Janssen JP, Linnaluoto M. Remote monitoring of implantable cardioverter defibrillator patients: a safe, time-saving, and cost-effective means for follow-up. Europace. 2008;10: 1145-51.

25. Halimi F, Cantù F; European Heart Rhythm Association (EHRA) Scientific Initiatives Committee (SIC). Remote monitoring for active cardiovascular implantable electronic devices; a European survey. Europace. 2010;12:1778-80.

26. Elsner CH, Sommer P, Piorkowski C, Taborsky M, Neuser H, Bytesnik J, et al. A prospective multicenter comparison trial of home monitoring against regular follow-up in MADIT II patients: additional visits and cost impact. Comput Cardiol. 2006;33:241-4.

27. Fauchier L, Sadoul N, Kouakam C, Briand F, Chauvin M, Babuty D, et al. Potential cost savings by telemedicine-assited long-term care of implantable cardioverter defibrillator recipients. Pacing Clin Electrophysiol. 2005;28 Suppl 1:255-9. 